\title{
INVESTIGACIONES
}

\section{Maestros que investigan: un estatus aún en disputa*}

\author{
Teachers researching: A status still on dispute
}

\author{
Nicolás Aguilar-Forero ${ }^{a}$, Gary Cifuentes ${ }^{a}$ \\ ${ }^{a}$ Facultad de Educación, Universidad de los Andes, Colombia. \\ nj.aguilar1902@uniandes.edu.co,gcifuent@uniandes.edu.co
}

\begin{abstract}
Este trabajo parte de un estudio que caracterizó las prácticas de docentes investigadores, así como las características de las instituciones que promueven o limitan dicha investigación en Bogotá, Colombia. Con base en un estudio mixto de tipo exploratorio secuencial-concurrente, se analizó el modo en que las prácticas de los docentes que investigan están atravesadas por condiciones tanto institucionales como culturales. Sobre las primeras se identificaron barreras asociadas a la disponibilidad de tiempo y al poco acompañamiento interno y externo para investigar, escribir y socializar las investigaciones realizadas. Respecto a la dimensión cultural, se encontró que el estatus de maestro investigador debe aún ser defendido en las instituciones educativas, elemento que merece especial reflexión dado el aumento creciente de maestros en (o con) formación posgradual que investigan en Bogotá. Este trabajo puede ser relevante para investigadores en educación, tomadores de decisiones en políticas de formación docente y maestros del sector público.
\end{abstract}

Palabras clave: investigación educativa, condiciones institucionales, formación docente.

\section{ABSTRACT}

This work is based on a study that characterized the research practices of teachers, as well as the characteristics of the institutions that promote or limit such research in Bogotá, Colombia. Based on a mixed sequential-concurrent exploratory study, we analyzed the way in which the practices of investigating teachers are traversed by both institutional and cultural conditions. On the former, barriers associated with time availability and little internal and external accompaniment were identified to investigate, write and socialize the research carried out. Regarding the cultural dimension, it was found that the status of a teacher-researcher must still be defended in educational institutions, an element that deserves special reflection given the increasing number of teachers in (or with) postgraduate training who develop researches in Bogotá. This work may be relevant for researchers in education, decision makers in teacher training policies and public sector teachers.

Key words: educational research, institutional conditions, initial teacher education.

\footnotetext{
* Este estudio fue financiado por el Instituto para la Investigación Educativa y el Desarrollo Pedagógico - IDEP. El IDEP es un establecimiento público de Bogotá, Colombia, que promueve y gestiona la investigación, la innovación y el seguimiento a la política educativa de la ciudad. La investigación fue realizada por un equipo de la Facultad de Educación de la Universidad de los Andes de Bogotá, con el apoyo del Centro de Evaluación de esta misma universidad.
} 


\section{INTRODUCCIÓN}

En el marco del Plan Sectorial de Educación 2016-2020 y de su apuesta por construir una ciudad educadora, la ciudad de Bogotá viene impulsando el reconocimiento y cualificación de sus maestros y maestras a través de distintas estrategias de fomento a la investigación e innovación en sus prácticas educativas. En ese contexto, el Instituto para la Investigación Educativa y el Desarrollo Pedagógico - IDEP realiza en 2019 el estudio Características individuales e institucionales que promueven la investigación y la innovación educativa en el Distrito Capital, el cual buscó responder, entre otras preguntas, las siguientes: ¿cuál es el perfil general que caracteriza a los docentes que promueven la investigación y la innovación educativa? ¿Cuáles son las prácticas de los maestros que están asociadas con dicha promoción?

Este escrito se centra en los hallazgos referidos a las características de docentes investigadores y a las condiciones que limitan sus prácticas de investigación. Lo anterior por ser este un tema que, como se verá, aún genera disputas y controversias en la ciudad de Bogotá. A continuación, se presenta el marco que soportó conceptualmente esta investigación. Luego se expone la estrategia metodológica basada en un diseño mixto y, para terminar, se resaltan hallazgos críticos que son relevantes tanto para investigadores en educación, como para tomadores de decisiones en el ámbito de la formación docente.

\section{MAESTRO INTELECTUAL VERSUS MAESTRO INVESTIGADOR: APORTES DESDE LA LITERATURA}

Pensar el maestro como intelectual implica abordar discusiones que se remontan al oficio del maestro y al lugar social y epistemológico que ocupa la práctica pedagógica. Como explicó Zuluaga (1999), la práctica pedagógica es un saber que posee su propio sujeto históricamente definido: el maestro. Un maestro que, a su vez, es un portador y generador de saberes. Sin embargo "tanto la condición de la pedagogía entre las disciplinas como la condición del maestro entre los intelectuales, son subalternas" (p. 13). En este sentido, la pedagogía se ha venido atomizando en el discurso de las ciencias de la educación, las cuales se han repartido su objeto de estudio, la práctica pedagógica, marginando a la pedagogía como saber y como disciplina. En paralelo, los maestros han venido perdiendo autonomía sobre su saber, el cual ha sido raptado por otras ciencias y otros intelectuales, y han venido asumiendo un rol instrumental (más ligado a un oficio metodológico) en detrimento de su rol como sujetos de saber pedagógico (ligado a un oficio intelectual) (Herrera y Martínez, 2018).

Abordar esta discusión conlleva necesariamente recuperar los aportes del movimiento pedagógico colombiano que, desde los años 80, reaccionó frente al proceso de curricularización vivido a lo largo del siglo XX "que convirtió al maestro en depositario de saberes y de modelos de enseñanza que repetía mecánicamente, los cuales devolvía a los alumnos mediante la instrucción" (Mejía, 2005, p. 7). Este movimiento articuló varios procesos divergentes, que:

en términos militantes nombraríamos como «la lucha del magisterio por el reconocimiento intelectual de su oficio», en términos analíticos podemos llamar «la reconfiguración de la subjetividad del maestro como productor de saber pedagógico», 
y en términos políticos nombramos como la transfiguración de su subjetividad política, pasando de autorepresentarse como un «sujeto social oprimido» entre otros, a reconocerse como un trabajador intelectual o de la cultura, que ha sido relegado a la condición de subalternidad intelectual por un régimen (epistemológico-político) de saber (Saldarriaga y Vargas, 2015, p. 314).

Aunque la reivindicación del maestro como intelectual y sujeto de saber pedagógico sigue vigente luego de 40 años de la emergencia del movimiento pedagógico en Colombia, hoy se enfrenta a nuevos desafíos y controversias: 1) la "evaluación de desempeño" que constriñe las prácticas de las nuevas generaciones de maestros y las ata a tecnicismos administrativas y a la voluntad de directivos docentes; 2) la desprofesionalización de la profesión docente cuyo síntoma es la admisión al escalafón de profesionales sin formación pedagógica (Saldarriaga y Vargas, 2015); 3) la generalización del rol de docenteinvestigador asociado a lógicas impuestas ya no solo desde el Estado, sino desde el mercado del conocimiento global: publicación en revistas indexadas, citación por parte de pares, rankings de investigadores y productivismo académico, que instrumentaliza y despolitiza a los docentes (Aguilar Soto, 2017a). Ello ha llevado a recuperar el estatus de maestro intelectual por encima del de docente-investigador, e incluso a posicionar la categoría de "no investigador" (como también la de revistas "no indexadas") como alternativa:

Como categoría de resistencia, ser no investigador no posee connotación de negación, pues no pone de relieve condición alguna de inferioridad. (...) [Expresa] un nosotros que reúne a investigadores y no investigadores que no renuncian a la publicación de textos de las más altas calidades académicas, pero que no lo hacen para satisfacer los requerimientos de la razón de Estado, sino de acuerdo con las exigencias que responden a principios de autorregulación de las comunidades (Aguilar Soto, 2017b, p. 82).

En este marco, sin embargo, si bien hay un sentido dominante de la investigación asociado al capitalismo cognitivo que está muy arraigado en las universidades, las prácticas de investigación educativa de los maestros son complejas, diversas y no se pueden reducir a este. La investigación educativa, como proceso de generación de conocimientos sobre temas educativos y pedagógicos, puede tener distintas finalidades: reflexionar sobre la propia práctica para transformarla, llevar a cabo un proceso metódico de recolección y sistematización de información según los criterios de las comunidades científicas, realizar un proyecto inscrito a una institución educativa o a una institución que financia el desarrollo de la ciencia, o realizar una producción escrita: publicaciones, ponencias para eventos académicos, entre otras (Barrios y Herrera, 2016; Cabra-Torres et al., 2013).

Asimismo, la investigación educativa se puede desarrollar desde diferentes enfoques epistemológicos y estrategias metodológicas, y depende en buena medida del reconocimiento (y autorreconocimiento) del maestro como investigador. Así, depende de que el maestro conciba su práctica como un espacio que trasciende los procesos de enseñanza y de aprendizaje, y reconozca "que en ella también se genera conocimiento, un conocimiento que se legitima por estar inmerso en la situación educativa" (Barrios y Herrera, 2016, p. 43). Esta concepción del maestro como investigador está mediada por diferentes variables, entre las cuales se encuentran las motivaciones y la formación posgradual. 
Con respecto a las motivaciones, estas pueden ser muy diversas y estar relacionadas tanto con factores extrínsecos como intrínsecos. Los primeros se asocian a la investigación promovida por actores externos (universidades, gobierno local o nacional, fundaciones, ONG, entidades privadas del sector educativo, etc.) que, a través de convocatorias, premios, apoyos y demás incentivos, fomentan la investigación de los maestros. Por su parte, las motivaciones intrínsecas son las que surgen de la necesidad o interés del maestro por visibilizar sus experiencias educativas, generar intercambios y aprendizajes en otros maestros, resolver problemas de su contexto inmediato o transformar prácticas y contextos educativos. Desde esta última línea, la investigación está ligada a una apuesta del docente por la liberación y la transformación social, que repercute en la concepción y vivencia de la escuela. Se trata de la búsqueda por construir "una escuela otra" en la que el estudiante pueda ser un sujeto pensante y crítico, opuesto a la subordinación utilitaria, tecnócrata y transmisora de conocimiento (Cadena et al., 2018).

A propósito de la formación posgradual, resulta interesante que buena parte de la investigación que realizan los docentes esté relacionada con su participación en programas de formación posgradual, que como mencionan Barrios y Herrera (2016), promueven que el docente sea percibido y se perciba a sí mismo como un agente de cambio que reflexiona e incide directamente sobre su quehacer, lo cual puede facilitar que se convierta en un docente que investiga. La formación posgradual también puede incidir en la superación de las percepciones negativas por parte de los docentes frente a la investigación, entre las que se han identificado las siguientes: considerar la investigación como un conocimiento teórico alejado de la práctica, relacionarla con estudios poco aplicables en ciertos contextos o asociarla a textos difíciles de comprender (IDEP, 2018; Guevara, 2017; Perines y Murillo, 2017; Murillo, Perines y Lomba, 2017).

Estas percepciones suelen estar influidas por el acercamiento que han tenido los maestros a la investigación educativa. Así, los docentes que en su desarrollo profesional han tenido un contacto con la investigación pueden ser más proclives a realizar investigaciones sobre y desde sus prácticas educativas, e incluso a concebir la investigación como estrategia pedagógica (Mejía y Manjarrés, 2011). Dicha cercanía a la investigación es promovida desde los programas de educación superior, aunque algunos estudios señalan que se promueve o aprovecha más en la formación posgradual que en la formación inicial de maestros (Perines y Murillo, 2017; Murillo, Perines y Lomba, 2017; Acosta et al., 2019).

Pero más allá de la educación formal está el reconocimiento social y la autopercepción del docente como investigador, como intelectual, o como ambos, sin que para muchos exista una distinción u oposición entre estos. Lo cierto es que, en cualquier caso, el estatus de maestro intelectual o investigador implica abandonar "su constitución como portador y transmisor de saber para convertirse en productor" (Mejía y Manjarrés, 2011, p. 145) y en agente de construcción pedagógica, educativa y social.

\section{ESTRATEGIA METODOLÓGICA}

La estrategia metodológica implementada se basó en un diseño mixto. En este tipo de estudios se integran métodos cualitativos y cuantitativos a partir de un diseño metodológico en el que se combinan distintas técnicas de recopilación de datos y procedimientos de análisis y/o inferencia. Los diseños mixtos tienen la virtud de ofrecer gran variedad de 
datos y perspectivas de los resultados, como la frecuencia, amplitud y generalización (cuantitativa), y la profundidad, complejidad y comprensión (cualitativa) (Creswell, 2013). Así, gracias a este diseño se logra integrar tanto la dimensión causal y explicativa (enfoque positivista), como la dimensión interpretativa o comprensiva (enfoque hermenéutico).

Específicamente, la estrategia metodológica que se implementó fue un diseño mixto de tipo exploratorio secuencial-concurrente. El estudio exploratorio es particularmente útil pues permite conocer o profundizar sobre un tema o fenómeno poco estudiado, a diferencia del estudio explicativo que busca comprender la causa o el porqué de un fenómeno (Hernández-Sampieri, Fernández y Baptista, 2010). Por su parte, mientras que la concurrencia implica que los componentes cuantitativo y cualitativo se implementan simultáneamente y se mantienen independientes en términos de recolección y análisis, en la secuencialidad hay una relación de interdependencia entre ambos componentes, en la medida que los resultados de una fase influyen en las decisiones de la etapa siguiente (Hernández-Sampieri, Fernández y Baptista, 2010).

En este sentido, la primera fase del estudio, secuencial, tuvo un carácter cualitativo exploratorio. Para esta primera fase no solo se recolectaron y analizaron fuentes secundarias, esto es, bibliografía especializada sobre la investigación educativa, sino que se reconoció el lugar de las redes y colectivos de maestros de la ciudad de Bogotá. Esto supuso no solo entrevistar a expertos en la academia para que se pronunciaran sobre el maestro investigador y la práctica misma que supone investigar en el campo educativo, sino realizar entrevistas y participar de encuentros en los que estas redes y colectivos enunciaban un discurso sobre el ethos del maestro que investiga. Con esta información se consolidó el marco teórico-metodológico, en el que se definieron las categorías de análisis de los principales constructos del estudio, que fueron la base para el diseño de los instrumentos cuantitativos y cualitativos de la siguiente fase.

En la segunda fase, concurrente, se trabajó de forma paralela e independiente el componente cuantitativo y cualitativo; en el primero de ellos se diseñaron, validaron y aplicaron dos cuestionarios en línea, uno diligenciado por 280 directivos y otro diligenciado por 4.572 docentes. Adicionalmente se realizó un muestreo probabilístico, bietápico y estratificado, para el cual se utilizaron diferentes bases de datos suministradas por el IDEP y la Oficina Asesora de Planeación de la Secretaría de Educación de Bogotá. Así, a través de dos tipos de aplicación, una censal y otra controlada, se buscó consolidar información relevante sobre las características y prácticas en investigación e innovación en la ciudad.

En esta segunda fase, en el componente cualitativo, se siguieron los planteamientos de Yin (2014) y Stake (1999) para poner en marcha un estudio de caso comparado. Para ello, con base en seis bases de datos proporcionadas por el IDEP, se seleccionaron ocho instituciones educativas luego de su clasificarlas en dos grandes grupos: aquellas que cumplían con al menos tres de los cuatro criterios explicados a continuación y aquellas que no cumplían con ninguno:

- Están clasificadas en A por sus altas puntuaciones en las pruebas de Estado (SABER $\left.3^{\circ}, 5^{\circ}, 9^{\circ}\right)$ para las áreas de matemáticas, ciencias y lenguaje.

- Cuentan con maestros vinculados a colectivos, redes o semilleros de investigación.

- Alguno de sus maestros ha sido nominado o ha ganado un Premio a la investigación o innovación educativa promovido por la Secretaría de Educación y el IDEP.

- Alguno de sus docentes ha presentado ponencias en eventos nacionales o internacionales. 
Entre los dos grupos de instituciones (las que cumplían con más de tres de estos cuatro criterios y las que no cumplían con ninguno) se seleccionaron ocho instituciones que se encontraban en los polos más opuestos según dichos criterios. La aproximación a cada institución educativa implicó la realización de entrevistas semiestructuradas con directivos y con docentes (16 en total) y un análisis de contenido del Proyecto Educativo Institucional de las instituciones seleccionadas. Todo lo anterior con el propósito comprender y profundizar en el fenómeno de estudio. Para efectos del presente artículo, se exponen a continuación los resultados relacionados con las características de docentes investigadores y las condiciones que limitan sus prácticas de investigación.

\section{RESULTADOS}

\subsection{CARACTERÍSTICAS DE DOCENTES INVESTIGADORES}

Una forma de caracterizar a los docentes investigadores es a partir de lo que piensan y lo que hacen, es decir, de sus concepciones y prácticas. Sobre las concepciones, se identificó que, para más de la mitad de los docentes encuestados en la ciudad de Bogotá, la investigación educativa es una forma de "reflexionar sobre la práctica docente para transformarla" $(57.4 \%)$. Sin embargo, para otros tiene otros significados, como se presenta en el siguiente gráfico:

Gráfico 1. Concepciones de los docentes sobre investigación

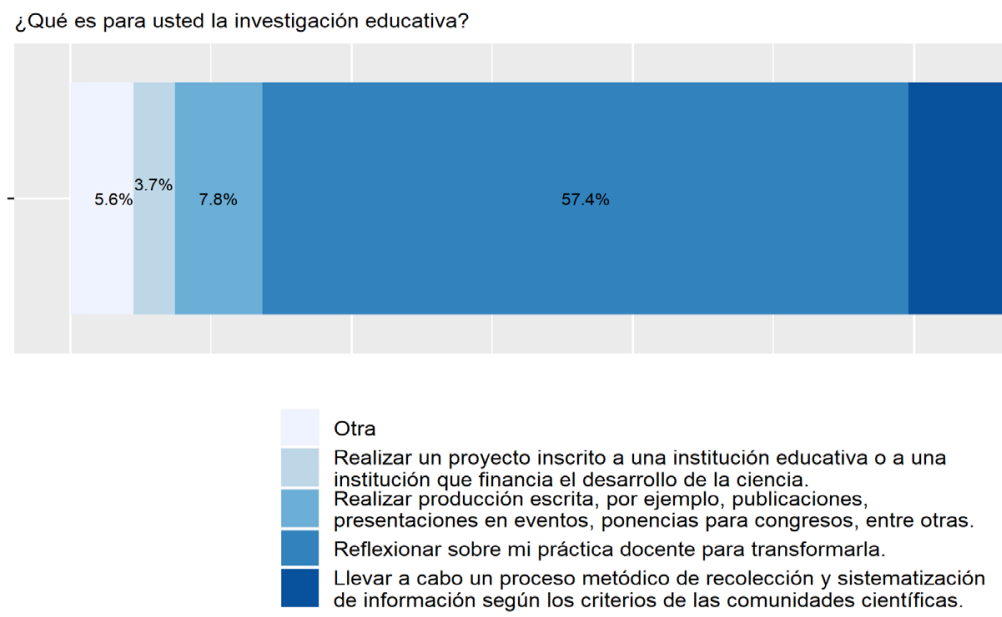

Fuente. Elaboración propia. Equipo de investigación - Facultad de Educación y Centro de Evaluación de la Universidad de los Andes de Bogotá. 
El énfasis de las respuestas anteriores en la investigación como medio para transformar la práctica docente fue también evidente en el trabajo cualitativo. Una de las docentes investigadoras entrevistada, cuya particularidad es que investiga en primaria, señaló que su principal motivación para investigar ha sido modificar su práctica pedagógica, frente a la necesidad que encontró de hacerlo cuando una niña con síndrome de Down ingresó como estudiante a su aula. Ello la lleva a cuestionar su actuar como docente, a identificar la falta de apoyos institucionales para atender a estudiantes con necesidades especiales y a investigar para poder crear el mejor ambiente educativo posible para ella y para los demás estudiantes de su clase:

Empecé a investigar para visibilizar a Catherine [nombre inventado por los autores para preservar la confidencialidad] y a los demás niños. De hecho, teníamos otros niños de inclusión. Si el colegio tiene más de 20 ahí sí son visibles y hay que mandar apoyo, pero si no hay 20 miren a ver qué hacen. Por eso yo escribo la primera ponencia con el trabajo con Catherine en el foro distrital de ese año, hace 5 años (...) A mí se me abre el mundo con este trabajo porque no es solo ella, la niña de inclusión, es Juan Pablo, es Pedro, es Alejandra, porque finalmente cada niño trae su propia historia de vida, su propia condición de vida. Uno dice: aparentemente los otros "son normales", pero esta niña viene de padres separados, este otro viene de la pobreza, este otro no desayunó hoy. Finalmente, cada niño tiene una condición de vida que a veces la escuela homogeniza, pero cada uno es tan diferente, muchas veces nos damos cuenta de que la práctica pedagógica es igual para todos, y si vamos a hablar de la práctica evaluativa ¿Por qué se evalúan a todos por lo mismo? Entonces desde ese momento yo empiezo a hacer un ejercicio distinto, de poner en la práctica la teoría. (Entrevista docente investigadora, 2 de agosto de 2019).

Con respecto a las concepciones, también se halló que los docentes que investigan valoran positivamente la investigación educativa. En efecto, para el $95.1 \%$ de encuestados la investigación "favorece el mejoramiento de las prácticas educativas" y para $93.7 \%$ es "necesaria para fortalecer los procesos pedagógicos de la institución educativa". Asimismo, el $90 \%$ considera que la investigación educativa "debería ser realizada por docentes y directivos docentes", frente a un $10 \%$ para el que "es necesaria, pero no cuando es realizada por docentes o por directivos docentes". Este último dato coincide con la posición de los docentes que pertenecen a redes o colectivos de maestros que fomentan la investigación, para quienes es muy importante la investigación que realizan los docentes, o que se realiza desde la institución y no solamente sobre la institución:

Hay dos perspectivas, las investigaciones que se hacen sobre la escuela y las investigaciones que se hacen desde la escuela. Desde la escuela es muy poco lo que se ha hecho porque generalmente se hace desde afuera, son los didactas, las universidades, los que investigan y nos dicen a nosotros lo que tenemos que hacer. Pero se ha desarrollado muchísimo conocimiento desde los profesores de colegio, por ejemplo, sobre la forma de enseñar ciencias, de enseñar física de otra manera, que puede ser muy importante pero que no está rastreado. (Docente que pertenece a una red de maestros investigadores, 2 de mayo de 2019) 
Sobre las prácticas de investigación educativa, se identificó que los temas más investigados por los maestros que investigan giran en torno a las didácticas de disciplinas específicas (lenguaje, matemáticas, artes, ciencias sociales, tecnología, ciencias naturales, etc.) (61.9\%), seguido de "transversalidad en las áreas" (49\%) y "tecnologías de la información y la comunicación (TIC) aplicadas al aprendizaje de los estudiantes" $(47.2 \%)$ :

Gráfico 2. Temáticas sobre las que investigan los docentes

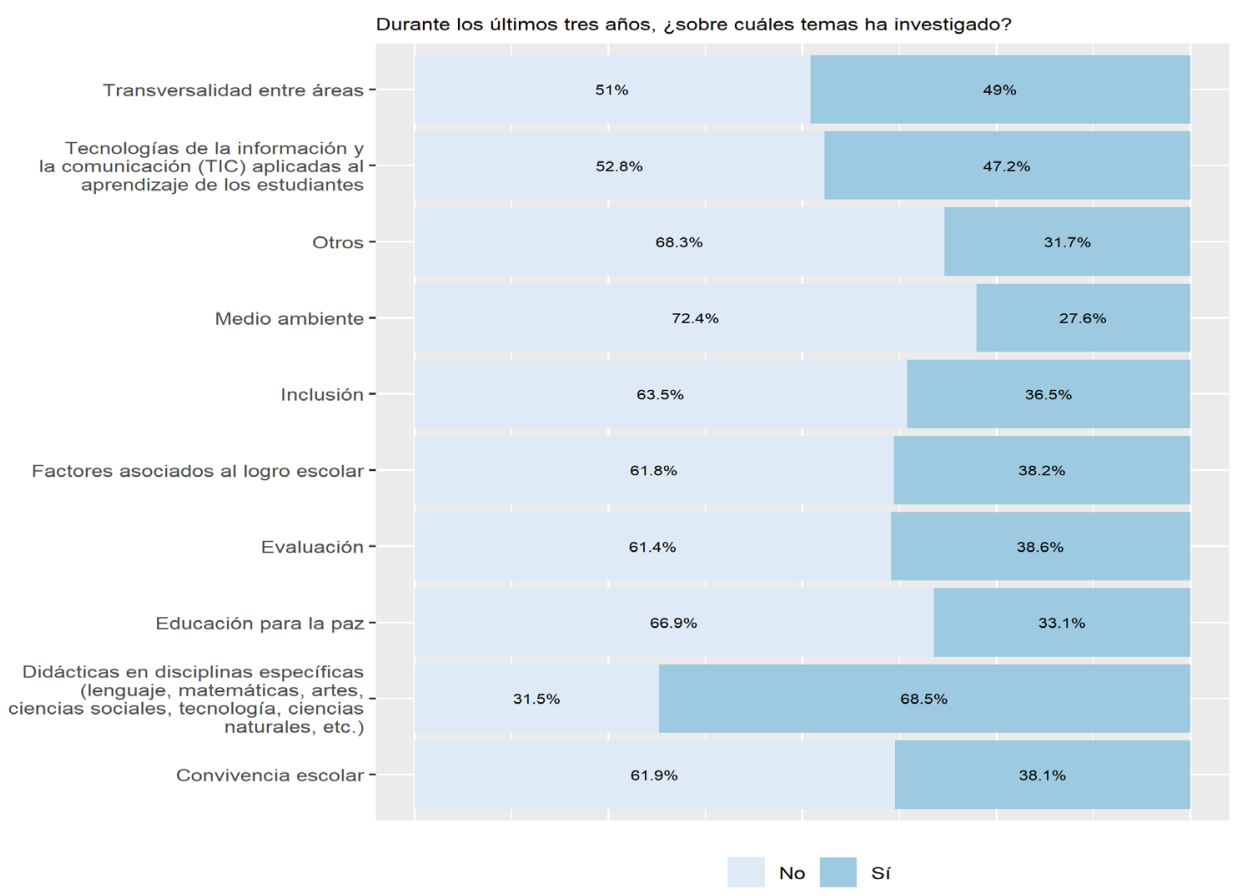

Fuente. Elaboración propia. Equipo de investigación - Facultad de Educación y Centro de Evaluación de la Universidad de los Andes de Bogotá.

Asimismo, las estrategias metodológicas más utilizadas al momento de investigar son la investigación-acción o Investigación Acción Participativa (IAP) (78.6\%) y la sistematización de experiencias $(69 \%)$. Las menos utilizadas son la investigación arqueológica y genealógica (6.3\%), la estadística inferencial (12.7\%) y el método científico (experimental y cuasi experimental) $(28.4 \%)$. Estos resultados evidencian la preferencia de los docentes por estrategias metodológicas propias del enfoque crítico de la investigación social, lo cual es coherente con sus motivaciones al momento de investigar (reflexionar sobre la práctica para transformarla). Lo anterior se pudo identificar también en el estudio de caso. Una de las docentes entrevistada lo expresó en los siguientes términos, vinculando su manera de investigar con la formación recibida en su maestría: 
La maestría sí fue para mí una herramienta maravillosa, no solo por la calidad de los maestros y de lo que nos exigían en términos de escribir y de leer, sino por el ejercicio que se hace de sistematizar la experiencia. Fue ahí cuando yo hago el ejercicio de sistematizar la experiencia del proyecto de aula lúdica que yo manejaba en la Sede A. Este ejercicio de sistematización de experiencias me permite ver cómo desde la investigación popular hay unos elementos muy importantes, y a mí se me hace que este ejercicio de sistematización se acomoda muchísimo a la escuela porque permite escuchar las voces de los actores. (Entrevista docente investigadora, 2 de agosto de 2019).

Los enfoques y posicionamientos críticos que se evidencian tanto en las concepciones como en las estrategias metodológicas preferidas por los maestros investigadores repercuten también en sus prácticas de aula. En efecto, las maestras y maestros que investigan no solo lo hacen sobre sus prácticas pedagógicas, pues también promueven la investigación de sus estudiantes y, por consiguiente, utilizan la investigación como estrategia pedagógica:

En mi clase, con la investigación, empezamos a tratar de fortalecer esa parte cooperativa en el desarrollo de un proyecto relacionado con alguno de los ejes temáticos que estemos trabajando. Cuando tienen ya la pregunta-problema y hemos revisado el marco teórico les digo: "Nos vamos, ¿qué tema tiene usted?" biotecnología del futuro, y dicen "eso qué es, yo a quién voy a entrevistar". Entonces así fue, les dije "¿a quién le interesa estudiar, analizar o priorizar líneas de investigación en biotecnología del futuro?". No conocían de Colciencias, no conocían de Corpoica, pues nada, "vamos a escribir a estas personas para ir a entrevistarlas y saber ellos qué piensan". (Entrevista docente investigadora, 13 de junio de 2019).

\subsection{CONDICIONES QUE DIFICULTAN LA INVESTIGACIÓN DE LOS DOCENTES}

Para la mayoría de los docentes encuestados la principal razón por la cual no investigan es la falta de tiempo. Alrededor del $73 \%$ de docentes dedica poco tiempo, menos de 3 horas semanales, a registrar datos sobre su práctica cotidiana como docente, a reflexionar sobre ella (más allá del tiempo en el que se reflexiona para planear clases) o a analizar los resultados relacionados con sus proyectos de aula. La mayor parte del tiempo a la semana la invierten corrigiendo trabajos y planeando clases (situación que sin duda se complejiza más si se cuenta con grupos muy numerosos de estudiantes por aula de clase, lo cual demanda más tiempo y trabajo por parte de los docentes).

Esta barrera, por supuesto, no depende solo del "buen o mal" manejo del tiempo de los docentes, como si se tratara de una decisión netamente individual, sino que depende de condiciones institucionales. Por ejemplo, al indagar acerca del grado de acuerdo frente a un conjunto de afirmaciones referidas a la institución en la que se trabajan, los resultados sobre las barreras asociadas a la disponibilidad de tiempo fueron los siguientes: 
Gráfico 3. Barreras asociadas a la disponibilidad de tiempo

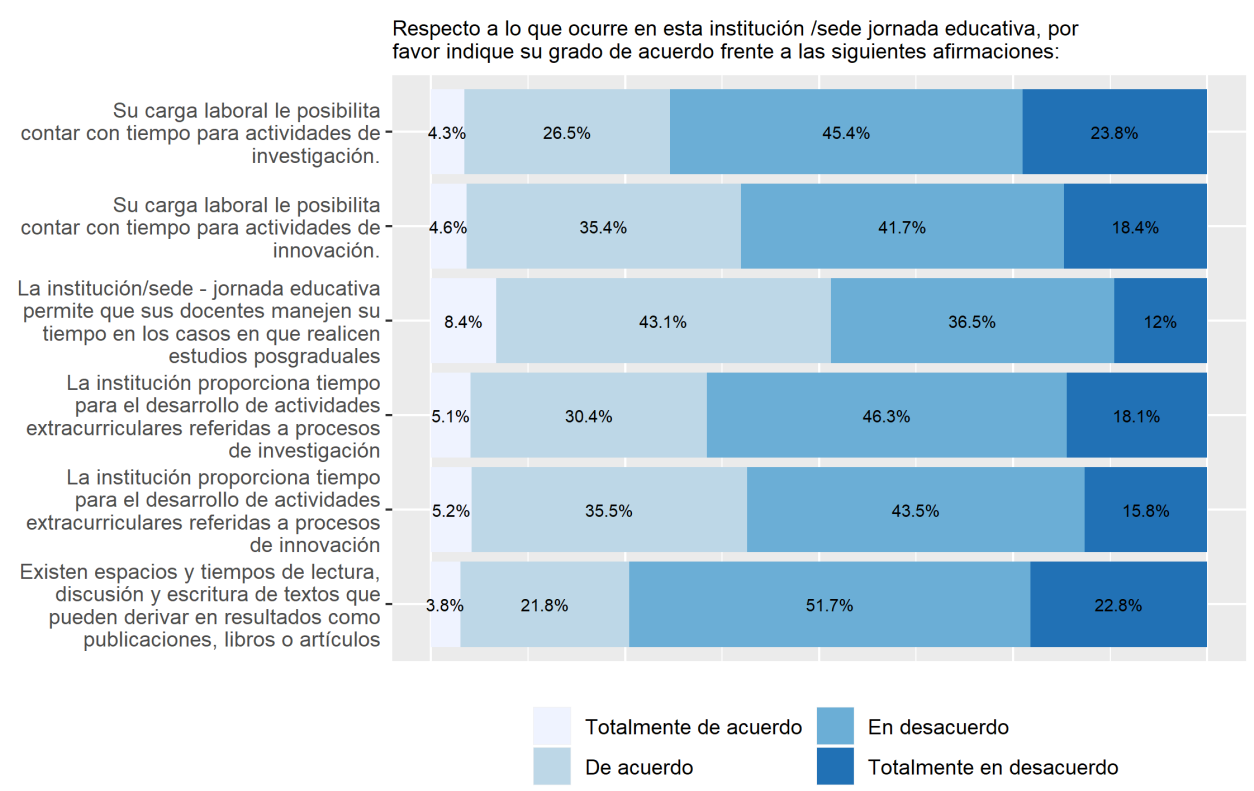

Fuente. Elaboración propia. Equipo de investigación - Facultad de Educación y Centro de Evaluación de la Universidad de los Andes de Bogotá.

Pero más allá de la disponibilidad de tiempo, desde el análisis de casos se pudieron identificar algunas otras condiciones que dificultan la investigación de los maestros. Una de ellas es el poco acompañamiento a los procesos de escritura y publicación. Como señaló una de las maestras entrevistada: "a mí me gusta escribir y sistematizar, pero no he podido hacer mi doctorado porque me han pedido el capítulo de un libro o un libro completo y no he podido hacerlo (...) yo quiero que un profesor pueda decir 'yo quiero escribir un libro' y que sepa a dónde ir y cuál es la ruta para hacerlo" (Entrevista docente investigadora, 13 de junio de 2019).

La falta de formación o entrenamiento en habilidades necesarias para la investigación, como la escritura, hace que la redacción de las experiencias y la generación de productos de investigación recaiga en uno o dos docentes que terminan sobrecargados de trabajo. Asî lo señaló una de las entrevistadas a propósito de la escritura de un informe de resultados de investigación:

Lo hemos intentado escribir desde hace rato, pero quien lo escribe soy yo (...) está el aporte de todas, pero finalmente la única que escribe soy yo, y por eso yo considero que esa es una falencia en ese momento porque en realidad sería importante poder vincularlas a todas en un ejercicio de escritura. (Entrevista docente investigadora, 2 de agosto de 2019). 
Esta maestra de primera infancia (e investigadora sobre primera infancia), identifica otro elemento que merece toda la consideración y que está relacionado con la percepción social del docente investigador: el estatus de maestro investigador es algo que aún tiene que disputarse en la sociedad, pero existen brechas en diferentes niveles educativos al punto que, en el caso de primera infancia, resulta aún más difícil asumir y posicionar dicho estatus:

Hablar del maestro como investigador es también hablar del maestro de primera infancia como investigador, como un productor de saber pedagógico. Porque históricamente el maestro de primera infancia, las maestras de transición, "son muy lindas, ellas cantan, son muy hábiles, hacen cosas muy lindas", pero la maestra de primera infancia si no habla argumentando es invisible. Por eso queremos que a las maestras de primera infancia se nos vea como creadoras de saberes e investigadoras permanentes (Entrevista docente investigadora, 2 de agosto de 2019).

Para ello, el tema de los tiempos, permisos y apoyos internos y externos para investigar resulta crucial. De hecho, como resultado de los frágiles apoyos institucionales (tiempos, espacios y permisos para participar de actividades extracurriculares relacionadas con la investigación), ciertos docentes interesados en sistematizar experiencias, investigar y escribir textos de investigación encuentran más oportunidades para hacerlo por fuera de la institución, como por ejemplo de la mano de las universidades (Cifuentes y Aguilar Forero, 2019). Así lo señaló una maestra investigadora, con formación doctoral, enfatizando en la necesidad de mayores apoyos económicos:

Mi compromiso es mantenerme viva en mi ejercicio investigativo. Después de que uno tiene un título de doctor si no pública e investiga muere. O sea, yo desde que obtuve mi título ejercí como doctora, siempre lo he hecho. Aquí en la Secretaría de Educación no, porque no me han dado la oportunidad de salir a otros espacios, pero sí en la universidad. No quiero decir con eso que aquí no lo haga, todo lo que yo investigo acá lo publico, pero con recursos de la universidad y no de la Secretaría (...) Trabajo en la Universidad de la Salle que me financia todas las investigaciones que hago tanto en las escuelas rurales como en esta donde trabajo (Docente, caso de institución que promueve la investigación, 7 de junio de 2019).

Dos dificultades se avizoran en este caso y competen a la política de promoción de la formación posgradual de los docentes en la ciudad Bogotá. Por una parte, cuando no hay un apropiado acompañamiento y apoyo, incluso económico, a la producción académica de los docentes investigadores, son las universidades las que terminan beneficiándose del conocimiento generado desde los contextos escolares. Así lo señaló uno de los docentes que pertenece a una red de maestros que realiza investigaciones y que fue entrevistado en la primera fase de este estudio:

En el distrito hay docentes que hacen investigación y ¿esa investigación para dónde se va? Esa investigación que producen los maestros generalmente es arrastrada y cooptada por las instituciones de educación superior. Eso también hace que, por ejemplo, yo no vea al maestro de escuela como un productor, pues es la universidad la que produce, 
entonces termina de legitimar un modelo que invisibiliza. Yo también trabajo para la Universidad de la Sabana y la Pedagógica y por estar haciendo el doctorado, estoy vinculado a un grupo de investigación [universitario]. Pero (...) ¿al final quién se beneficia de todo? La que se beneficia más fuertemente es la universidad que es la que está mostrando producción. Y cuando nosotros queremos reconocer la producción de los maestros de escuela, pues no se ve institucionalmente. (Docente que pertenece a una red de maestros investigadores, 2 de mayo de 2019).

En estos casos hay una vinculación a instituciones de educación superior que se benefician de la producción académica de los docentes de escuela. Desde luego, ellos también se benefician de las universidades no solo por la visibilidad que pueda tener su producción, sino por los apoyos económicos y las condiciones favorables para ejercer su rol de maestros investigadores. El riesgo con ello es que, cuando las condiciones institucionales son más favorables para los docentes investigadores estando afuera en lugar de adentro de su institución educativa ¿Qué garantiza que quieran quedarse en esta en lugar de irse a las universidades en las que, de hecho, ya trabajan?:

Yo no quise irme del colegio, porque casi todos los que son doctores se van de la escuela a la universidad, porque no hay incentivos. Yo me quedé porque lo que he siempre pensado es que algún día puede generarse esa situación de trabajar con otros. Yo ya lo hago por mi cuenta, pero esto debe ser desde la Secretaría de Educación y desde donde haya las condiciones. (Docente, caso de institución que promueve la investigación, 7 de junio de 2019).

En este sentido, la segunda dificultad tiene que ver el rol asumido por los docentes con formación doctoral que, como en el ejemplo anterior, deciden quedarse en la institución educativa. Algunos rectores o directivos no saben qué hacer con este perfil de docente en su institución e incluso llegan a juzgarlos porque sus prácticas "no cambiaron" a pesar de sus estudios, porque no continuaron sus investigaciones o porque estas no tuvieron ningún efecto en la institución: "no sabemos de qué es su investigación, cumplieron con el requisito para graduarse, pero no trascendió, ni siquiera nos enteramos cuál fue el proyecto de investigación que se hizo o si lo podemos aplicar para el área, los compañeros o para toda la institución, entonces se queda ahí" (Directivo docente de institución que no promueve la investigación, 22 de agosto de 2019).

Visto así pareciera que la responsabilidad de que le investigación doctoral arroje frutos en la institución recayera exclusivamente en los maestros. No obstante, desde la perspectiva de estos el problema también tiene que ver con la apertura de los directivos y, en definitiva, con el reconocimiento social e institucional del maestro investigador que, como se señaló, sigue siendo un estatus aún en disputa:

Si el proceso de investigación no se articula a las dinámicas de la institución se piensa como innecesario. Pero con otro compañero de doctorado planteamos toda la reformulación del currículo a través de procesos de investigación hablando con la comunidad (...) nos dio que el colegio debería hacer una enseñanza basada en proyectos. Nosotros hemos intentado mostrarle eso a él [al rector]: "si usted nos dejara actuar, si nos dejara estar en otros escenarios, nosotros vamos a producir" (...) Otro 
problema es que no se tienen espacios. Entre las funciones del profesor de universidad hay un espacio que es investigativo. En el caso de nosotros no hay ese espacio, no está reconocido. Todo lo que nosotros hacemos en términos de investigación es trabajo por fuera de nuestras labores como docentes. (Docente que pertenece a una red de maestros investigadores, 2 de mayo de 2019)

\section{DISCUSIÓN Y CONCLUSIONES}

Este estudio permitió identificar un conjunto de características asociadas a los maestros que investigan en las instituciones educativas del distrito, así como ciertas condiciones institucionales y culturales que dificultan sus prácticas de investigación. Sobre los resultados, un elemento para discutir tiene que ver con la relación entre investigación y formación docente. Como se identificó en la evaluación de impacto del programa de formación docente en Bogotá (Acosta et al., 2019), existe una asimetría entre los logros individuales de los docentes y la capacidad institucional para aprovechar las ventajas de la formación posgradual. Asimismo, aunque los docentes valoran los aprendizajes que han obtenido en los programas de formación posgradual que ha cursado, aún se "requiere de una mayor articulación de los docentes formados con las dinámicas institucionales" (Acosta et al., 2019, p. 16).

Esta desarticulación y la falta de condiciones institucionales para aprovechar y potenciar el saber de las maestras y maestros con formación posgradual, con maestría o doctorado, conlleva un riesgo latente: al no encontrar en la escuela condiciones favorables para el desarrollo de su práctica pedagógica e intelectual, el maestro puede preferir alejarse de los contextos escolares y buscar mejores condiciones, por ejemplo, en contextos universitarios. En un momento en el que más del $60 \%$ de los docentes de Bogotá tiene maestría y hay más de 120 maestras y maestros con formación doctoral, promover incentivos y condiciones en las instituciones educativas para que estos puedan desarrollar sus prácticas de investigación resulta crucial, para así lograr que el conocimiento generado revierta y repercuta en estas.

Sin embargo, es importante considerar que ciertos incentivos no son, per se, positivos. Por ejemplo, la investigación que emana de la formación posgradual motivada por la promoción en el escalafón docente ${ }^{1}$ no siempre tiene los efectos esperados. Muchas investigaciones que parten de motivaciones extrínsecas "son forzadas por la necesidad, sobre todo porque los profes del nuevo escalafón ascendemos de esta manera, entonces necesitamos nuestra maestría, necesitamos nuestro doctorado para recibir un aumento salarial. Pero muchas de esas investigaciones se quedan ahî" (Entrevista docente investigador, 24 de mayo de 2019).

Por esta razón, además de las motivaciones extrínsecas, reconocer las motivaciones intrínsecas y generar apoyos institucionales a través de alianzas con otras instituciones, redes, organizaciones y entidades que acompañen los procesos de investigación, escritura y socialización de las investigaciones de los maestros, puede ser una clave relevante. No obstante, si bien ello puede contribuir a fortalecer el reconocimiento social del maestro como intelectual, es evidente que se requiere promover cambios no solo a nivel institucional sino cultural. Esto pasa, por ejemplo, por la formación de las futuras generaciones de

El estatuto 1278 de 2002 que reglamenta la profesión docente en Colombia incentiva la formación posgradual de los maestros (y por extensión la investigación que se promueven en los programas de maestría y doctorado) al conceder un incremento salarial de acuerdo con el nivel educativo alcanzado. 
maestros en las licenciaturas y Facultades de Educación, por el tipo de sujeto-maestro que se promueve en estas y por la disputa acerca del tipo de investigación que es relevante para la escuela y para la sociedad.

Sobre este punto, este estudio permitió identificar que tanto las concepciones de los maestros sobre investigación educativa como las metodologías ligadas a esta que más implementan (investigación-acción, IAP y sistematización de experiencias) están vinculadas con enfoques epistemológicos y teórico-políticos de corte crítico. Este dato es valioso, pues evidencia que estos enfoques críticos ocupan un lugar importante en los programas de formación docente. Sin embargo, no solo el espíritu crítico circula en las Facultades de Educación. Cabe alertar sobre el riesgo de importar en la escuela las lógicas universitarias de investigación, cada vez más orientadas a la publicación en cadena, a los rankings, puntos y demás "incentivos" asociados a ciertos desplazamientos: del "conoceraprender" al "conocer-adquirir", del conocimiento socialmente relevante al conocimiento socialmente legitimado por centros institucionalizados de saber, entre otros (Aguilar Soto, 2017a, p. 16). Recuperar y potenciar la condición de maestro intelectual e impulsar las prácticas de generación de conocimiento colaborativas, comunitarias y complementarias a las lógicas dominantes de investigación, resulta ahora, más que nunca, fundamental.

\section{REFERENCIAS BIBLIOGRÁFICAS}

Acosta, J., Castillo, M., Cortés, D., Gómez, M., González, J. I., López, A., Maluendas, A., Ortiz, J. G. \& Sandoval, M. (2019). Evaluación de impacto del programa de formación docente en Bogotá. Revista Educación Y Ciudad, 1(36), 93-104. https://doi.org/10.36737/01230425. v1.n36.2019.2136

Aguilar Soto, J. F. (2017a). III encuentro de socialización de experiencias educativas y prácticas pedagógicas en el contexto universitario. -Algunos temas de reflexión-. En J. F. Aguilar Soto (comp.), Memorias del Tercer Encuentro de Socialización de Experiencias Educativas y Prácticas Pedagógicas en el Contexto Universitario. Bogotá: Instituto de Estudios e Investigaciones Educativas.

Aguilar Soto, J. F. (2017b). Manifiesto del No Investigador. Revista Noria - Investigación Educativa, $1(1), 80-82$.

Barrios, D. y Herrera, J. (2016). Formación posgradual en investigación y profesión docente. Voces y Silencios: Revista Latinoamericana de Educación, 7(1), 32-63. DOI: http://dx.doi.org/10.18175/ VyS7.1.2016.03

Cabra-Torres, F., Herrera, J., Gaitán, C., Castañeda-Peña, H., Garzón, J. y Marín-Díaz, D. (2013). La investigación e innovación en la formación inicial de docentes. Aportes para la reflexión y el debate. Bogotá: Universidad Pontifica Javeriana.

Cadena et al. (Colectivo de Pensamiento Pedagógico Contemporáneo, 2018). Pensamiento pedagógico contemporáneo. Experiencias de transformación colectiva a pie de página. Bogotá: Editorial Magisterio.

Cifuentes, G. y Aguilar Forero, N. (2019). Condiciones institucionales para innovar e investigar: recomendaciones para su promoción en el Distrito Capital. Notas de Política en Educación No. 4 (ISSN 2619-4597).

Creswell, J. (2013). Research design: qualitative, quantitative and mixed methods approaches. Thousand Oaks, CA: Sage.

Guevara, J. (2017). La formación en los espacios de práctica docente: modos de transmisión del oficio. Estudios Pedagógicos, 43(2), 127-145. 
Hernández-Sampieri, R., Fernández Collado, C. \& Baptista Lucio, P. (2010). Metodología de la investigación (5a. ed.). México D.F.: McGraw-Hill.

Herrera, J. y Martínez, Á. (2018). El saber pedagógico como saber práctico. Pedagogía y Saberes, (49), 9-26.

IDEP. (2018). Coordinación de la propuesta conceptual y metodológica del Premio a la Investigación e Innovación educativa 2018 y Apoyo académico del proceso validación final de instrumentos y evaluación de propuestas presentadas a la XII versión del Premio a la Investigación e Innovación Educativa 2018. Bogotá: Institución para la Investigación Educativa y el Desarrollo Pedagógico.

Mejía, M. (2005). Los movimientos pedagógicos en tiempos de globalizaciones y contrarreforma educativa. Nodos y Nudos, 2(18), 4-19.

Mejía, M. y Manjarrés, M. (2011). La investigación como estrategia pedagógica. Una apuesta por construir pedagogías críticas en el siglo XXI. Praxis \& Saber, 2(4), 127-177. https://doi. org/10.19053/22160159.1127.

Murillo, F., Perines, H. y Lomba, L. (2017). La Comunicación de la Investigación Educativa. Una Aproximación a la Relación Entre la Investigación, su Difusión y la Práctica Docente. Profesorado, 21(3), 183-101.

Perines, H. y Murillo, J. (2017). Percepciones de los docentes en formación sobre la investigación educativa. Estudios pedagógicos, 43(1), 251- 268.

Saldarriaga, O. y Vargas, C. (2015). La configuración histórica del maestro público en Colombia: entre opresión y subalternidad, 1870-2002. Historia y Memoria de la Educación, 1(1), 287-318.

Stake, R. E. (1999). Investigación con estudio de casos. Madrid: Morata.

Yin, R. K. (2014). Case study research: Design and methods ( $5^{\text {th }}$ ed.). Thousand Oaks, CA: Sage.

Zuluaga, O. (1999). Pedagogía e historia: la historicidad de la pedagogía, la enseñanza, Un objeto de saber. Santa fe de Bogotá: Siglo del Hombre Editores. 
